\title{
The patterns and management of fracture patients under COVID-19 outbreak in China
}

\author{
Pei Yu", Chuanlong Wü, Chengyu Zhuang, Tingjun Ye, Yin Zhang, Jingfeng Liu, Lei Wang \\ Department of Orthopedics, Ruijin Hospital Affiliated to Shanghai Jiao Tong University School of Medicine, Shanghai, China \\ Contributions: (I) Conception and design: P Yu; (II) Administrative support: L Wang; (III) Provision of study materials or patients: P Yu, C Zhuang, T \\ Ye; (IV) Collection and assembly of data: C Wu, Y Zhang; (V) Data analysis and interpretation: P Yu, C Wu; (VI) Manuscript writing: All authors; (VII) \\ Final approval of manuscript: All authors. \\ \#These authors contributed equally to this work. \\ Correspondence to: Lei Wang. Department of Orthopedics, Ruijin Hospital Affiliated to Shanghai Jiao Tong University School of Medicine, Shanghai \\ 20025, China. Email: ray_wangs@hotmail.com.
}

Background: Currently, the perioperative care of fracture patients is compromised due to the outbreak of COVID-19 in China and the world. This study aims to assess the clinical features of fracture patients at our hospital during the COVID-19 outbreak and formulate the medical steps to ensure the effective treatment of fracture patients with minimal risk of infection to healthcare workers.

Methods: One hundred twelve patients with different fractures that were admitted to the orthopedics department of our hospital from January 24 to March 9 in 2020 were reviewed. Data including age, gender, injury location, admission time, operation time, discharge time were compared with fracture patients from the same period in 2019

Results: Compared to the same period in 2019, there is a $42 \%$ decrease in the number of fracture patients in 2020. Specifically, the incidences of forearm, thigh, hand, and foot fractures have increased during the COVID-19 outbreak, while other parts are less affected. The time from injury to hospitalization, the surgery wait time and time of discharge after surgery for patients with hip fractures were $2.9 \pm 7.1,2.0 \pm 1.7$ and $7.7 \pm$ 4.0 days respectively in 2019 , which changed to $2.0 \pm 5.0,4.5 \pm 4.0$ and $10.6 \pm 4.2$ days in 2020 . Following the orthopedic treatment regimen followed at our hospital, all patients had non-life-threatening limb fractures. Six patients were operated in a negative pressure room, and emergency screening was completed for six patients. No patients were positive for COVID-19, and all were discharged safely without infection or other serious complications.

Conclusions: Hip fractures are highly prevalent during this epidemic. However, mandatory screening delays surgery by more than 48 hours. The orthopedic department should prioritize screening of emergency patients to minimize the risk of infection among other patients and medical personnel.

Keywords: COVID-19; fracture; pattern; management

Submitted Apr 29, 2020. Accepted for publication Jul 04, 2020.

doi: 10.21037/atm-20-4174

View this article at: http://dx.doi.org/10.21037/atm-20-4174

\section{Introduction}

COVID-19 spread across China and the world and is, at present, a global pandemic $(1,2)$. The virus is transmitted through respiratory droplets, aerosols, and close contact with the infected patients, and the asymptomatic individuals are the main source of infection spread (3). The diagnosis of COVID-19 relies on the identification of the novel coronavirus genome by PCR or gene sequencing (4). A recent case study on 4,021 confirmed patients in China (diagnosed on January 26) showed that most were aged $30-65$ years $(71.45 \%)$, while another study reported that 


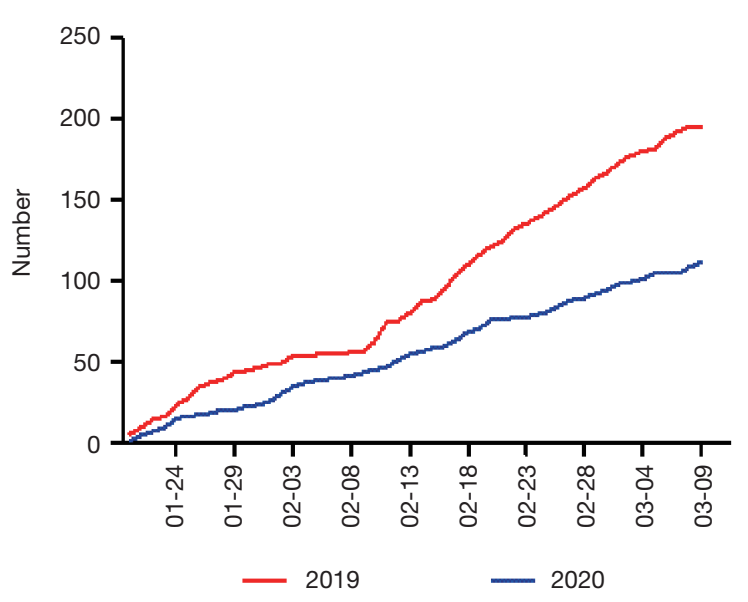

Figure 1 Changes in the number of patients admitted to orthopedics.

nearly half of the COVID-19 patients were over 50 years old (5). Most patients with light COVID-19 infection have a good prognosis, whereas severely infected patients exhibit dyspnea for more than a week after onset, acute respiratory distress syndrome, septic shock, acid-base imbalance, coagulation disorder and multiple organ failure (6-8).

The general susceptibility of the population to COVID-19 and considerable variations in the incubation period of the virus supplies new challenges to the diagnosis and treatment of orthopedic disorders. Fracture patients often do not comply due to swelling and pain, which prolongs the recovery of the joint function $(9,10)$. And, elderly patients that recuperate over a long period are highly susceptible to respiratory failure, urinary, and genital tract infection, stones, bedsores, body temperature disorders, deep vein thrombosis, and other complications. Timely surgical intervention and advanced mode analgesia can accelerate recovery and prevent the above complications (11).

However, perioperative care of fracture patients is compromised, because following standard medical procedures for fracture patients during the outbreak of COVID-19 can significantly increase the risk of infection among patients and healthcare workers. So, there is an urgent need to understand the current epidemiological characteristics and clinical manifestations of fractures in order to diagnose and treat them without increasing the risk of infection. In this study, we assessed the clinical features of fracture patients at our hospital during the COVID-19 outbreak and formulate the medical steps in order to ensure the effective treatment of fracture patients with minimal risk of infection to healthcare workers.

We present the following article in accordance with the STROBE reporting checklist (available at http://dx.doi. org/10.21037/atm-20-4174).

\section{Methods}

\section{Patients and data collection}

The patients admitted to the orthopedics department of our hospital for fracture were retrospectively reviewed from January 24 to March 9 in 2020.The fractures were classified based on Fracture and Dislocation Classification Compendium-2018 (AO/OTA classification) (12). Epidemiological data and treatment details were obtained from the chart review and discussed in a multidisciplinary team meeting. Other clinical information from the same period last year was also collected. This study was approved by the ethics committee of our hospital (No. KY2020-125). All procedures performed in this study involving human participants were in accordance with the Declaration of Helsinki (as revised in 2013). Because of the retrospective nature of the research, the requirement for informed consent was waived.

\section{Statistical analysis}

SPSS 17.0 software was used for statistical analysis. The measurement data were expressed as mean $\pm \mathrm{SD}$, and compared by the $t$-test, the category data were expressed as $\mathrm{n} / \%$ and compared by the Chi-square test or Fisher's exact test. A $\mathrm{P}<0.05$ was considered statistically significant.

\section{Results}

\section{Characteristics of orthopedic cases during the epidemic period}

A total of 112 orthopedic patients were admitted to the department of orthopedics at our hospital from January 24 to March 9 in 2020, while there were 196 orthopedic patients admitted in the same period in 2019, yielding a $42 \%$ decrease in admission (Figure 1). Following the AO classification (Table 1), there was no significant difference regarding the classification of fracture between 2019 and $2020(\mathrm{P}=0.218)$.

To be noted, there were 66 patients $(33.7 \%)$ with thigh fracture (type $31-34)$ in 2019 , and 51 patients (45.5\%) in 2020 , indicating thigh fracture was the top type of fracture 
Table 1 Characteristics of orthopedic cases in the epidemic period

\begin{tabular}{lcc}
\hline Fracture types & $2019(\mathrm{~N}=196)$ & $2020(\mathrm{~N}=112)$ \\
\hline Upper arm (11-16) (n/\%) & $28 / 14.3 \%$ & $9 / 8.0 \%$ \\
Forearm (2R2U) (n/\%) & $28 / 14.3 \%$ & $17 / 15.2 \%$ \\
Thighs (31-34) (n/\%) & $66 / 33.7 \%$ & $51 / 45.5 \%$ \\
Lower leg (41-44) (n/\%) & $40 / 20.5 \%$ & $21 / 18.8 \%$ \\
Vertebra (n/\%) & $22 / 11.2 \%$ & $6 / 5.4 \%$ \\
Pelvis and acetabulum (n/\%) & $2 / 1.0 \%$ & $0 / 0.0 \%$ \\
Hand $(\mathrm{n} / \%)$ & $3 / 1.5 \%$ & $2 / 1.8 \%$ \\
Foot $(\mathrm{n} / \%)$ & $7 / 3.6 \%$ & $6 / 5.4 \%$ \\
\hline
\end{tabular}

in both years.

\section{Characteristics of hip fractures cases in the epidemic period}

The disability and mortality rates of hip fractures are high, especially in elderly patients. We retrospectively analyzed the clinical data of 88 patients with hip fractures (50 cases in 2019 and 38 cases in 2020). There were 26 males and 24 females (average age $76.7 \pm 12.4$ years) who were admitted for hip fractures in 2019, and 12 male and 26 female patients (average age $77.3 \pm 14.9$ years in 2020 . The time from injury to hospitalization, the surgery wait time and time of discharge after surgery were respectively $2.9 \pm 6.4$, $2.0 \pm 1.5$ and $7.7 \pm 4.0$ days in 2019 , which changed to $2.0 \pm 5.2$ $(\mathrm{P}=0.47), 4.5 \pm 4.1(\mathrm{P}=0.00)$ and $10.6 \pm 4.3$ days $(\mathrm{P}<0.00)$ in 2020 (Table 2). These findings could be attributed to the more thorough screening and testing being conducted during the epidemic period to exclude the possibility of pneumonia.

\section{Treatment of hip fracture}

A total of 50 hip fracture patients admitted in 2019. There were 24 cases with Intertrochanteric fracture, who received proximal femoral nail anti-rotation (PFNAII) fixation. There were 26 cases with femoral neck fracture, among whom, 7 patients receiving cannulated screw fixation, 19 cases receiving hip arthroplasty. In 2020, there were 38 hip fracture patients admitted. There were 17 cases with Intertrochanteric fracture, who received PFNAIIfixation. And there were 21 cases with femoral neck fracture, among whom, 3 cases receiving cannulated screw fixation, and 18 cases receiving hip arthroplasty.

\section{Standard procedure in the orthopedic department during the epidemic}

The current guidelines stipulate that except serious/lifethreatening conditions, fracture patients should be treated for a limited period to ascertain coronavirus infection. For emergencies endangering life and limb function, such as vascular and nerve injury, amputated finger, and limb, large tissue defects, etc., the patients should be treated immediately and screened for novel coronavirus infection by pulmonary CT. Furthermore, the number of operations should be strictly controlled during the epidemic period, and the duration of operation should be planned following the surgical indications (13). I highly suspected or confirmed COVID-19 patients require emergency surgery, or if screening is not possible within the time frame, the operation should be performed in a room maintained at negative pressure, and the relevant personnel should follow appropriate protective steps. Furthermore, the patients should be kept in separate isolation wards until the screening.

Patients without life-threatening conditions should be triaged and screened by evaluating body temperature, respiratory symptoms, blood routine, and chest CT. Patients exhibiting fever with respiratory symptoms, fever without respiratory symptoms, but with previous exposure, bilateral lung lesions, decreased leukocyte and lymphocyte counts, respiratory symptoms without fever or fatigue, or double lung CT consistent with COVID-19 infection should be referred to the respiratory department for consultation. Patients with suspected COVID-19 infection should be temporarily admitted to the trauma surgical isolation ward, and then to a designated hospital if a positive diagnosis is confirmed by PCR from throat swabs samples (14). Patients 
Table 2 Characteristics of hip fractures cases in the epidemic period

\begin{tabular}{|c|c|c|c|c|}
\hline Characteristics & $2019(\mathrm{~N}=50)$ & $2020(N=38)$ & $\chi^{2} / t$ & $P$ value \\
\hline Male & $26 / 52.0 \%$ & $12 / 31.6 \%$ & & \\
\hline Female & $24 / 48.0 \%$ & $26 / 68.4 \%$ & 3.67 & 0.06 \\
\hline Age (y) & $76.7 \pm 12.4$ & $77.3 \pm 14.9$ & 0.19 & 0.85 \\
\hline \multicolumn{5}{|l|}{ Place of occurrence $(n / \%)$} \\
\hline Indoor & $24 / 48.0 \%$ & $25 / 65.8 \%$ & & \\
\hline Outdoor & $26 / 52.0 \%$ & $13 / 34.2 \%$ & 2.77 & 0.10 \\
\hline \multicolumn{5}{|l|}{ Fracture site $(\mathrm{n} / \%)$} \\
\hline Time from injury to hospitalization (d) & $2.9 \pm 6.4$ & $2.0 \pm 5.2$ & 0.73 & 0.47 \\
\hline Surgery wait time (d) & $2.0 \pm 1.5$ & $4.5 \pm 4.1$ & 2.71 & 0.00 \\
\hline Time of discharge after surgery (d) & $7.7 \pm 4.0$ & $10.6 \pm 4.3$ & 5.85 & $<0.00$ \\
\hline
\end{tabular}

that are scheduled for elective surgery and can be treated conservatively after evaluation should be observed at home. Remote diagnosis and treatment are also possible for nonemergency situations, and the patient can be operated once the epidemic is controlled. If surgery is necessary within a certain time limit, the patient should be screened for COVID-19 infection and transferred to a designated hospital if a positive diagnosis is confirmed. Patients that require re-visitations following a simple operation should be guided remotely to prolong the interval between re-visits as much as possible.

Routine blood examination and chest CT are recommended for regular admissions, and patients and their families with positive findings should be further screened to ensure that no COVID-19 cases are missed (15). Clinics lacking testing equipment should transfer suspected cases to designated hospitals at the earliest. Symptoms with manifestations like neo-coronal pneumonia should be found, and the possibility of other pneumonia pathogens and non-infectious diseases should be excluded. Suspected or confirmed COVID-19 cases should be reported immediately following national regulations, and transferred to a designated hospital once the patient is stabilized. Emergency surgery should be performed in designated hospitals with adequate protection facilities. Proper communication between the management and medical personnel is crucial to operating and the preparatory steps in isolation wards.

Following the orthopedic treatment regimen followed at our hospital (Figure 2), all patients had non-life-threatening limb fractures. Six patients were operated in a negative pressure room, and emergency screening was completed for six patients. None of the patients were positive for COVID-19, and all were discharged safely without infection or other serious complications.

\section{Indications for orthopedic surgery during the epidemic period}

During this epidemic, emergency surgery is recommended for patients with the proper physical constitution, unstable vital signs, progressive and aggravated injuries, and no possibility of non-surgical recuperation. Some of these situations are one, open lesions caused by high energy, severe bone, and soft tissue injuries. Two, severe spinal and spinal cord injuries affecting respiratory and cardiac function that can progress to incomplete paralysis. Three, joint dislocations and open reductions that cannot be manually fixed. Four, infectious lesions, such as incision and drainage of local infection focus. For patients with severe and critical neocoronal pneumonia combined with the above surgical indications, the focus should be on saving 


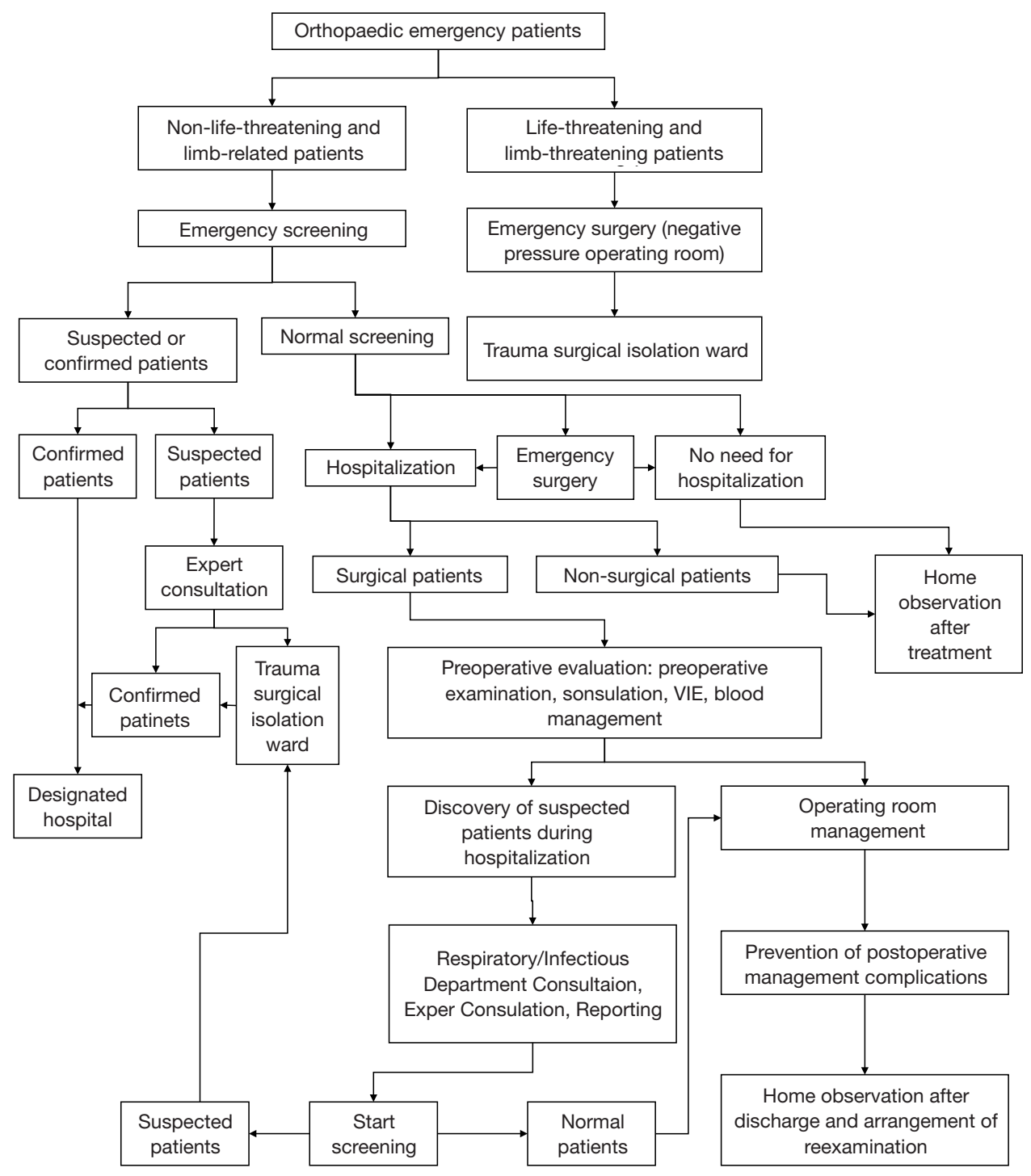

Figure 2 Diagnosis and treatment process of orthopedic emergency patients during the COVID-19 epidemic.

lives and then stabilizing the orthopedic condition, which can be followed by limited or elective surgery.

Non-surgical treatment of orthopedic disorders may cause severe complications including but not limited to (I) unstable spine fractures leading to incomplete paralysis that cannot be stabilized by traction, (II) aggressive bone and joint tumors that are recalcitrant to chemoradiotherapy, (III) moderate neurological dysfunction caused by cervical spondylosis and thoracic vertebral lesions, or severe cauda equina syndrome caused by lumbar disc herniation, (IV) unstable pelvic fractures with stable hemodynamics and lack of the urinary tract and other injuries, (V) improper resolution of limb and joint fractures without surgical reduction, and (VI) severe pain caused by various reasons. Furthermore, patients with new corona infection are often unresponsive to conservative treatment, whereas those with stable pneumonia can tolerate surgery.

\section{Intraoperative precautions}

For patients confirmed to be free of COVID-19, the routine surgical procedure should be followed. For suspected or confirmed COVID-19 patients, the number of people and equipment/supplies in the operating room should be 
minimized. If intraoperative fluoroscopy is needed, the technicians should wear protective clothing. Critically ill patients should be given respiratory and circulatory support to prevent complications under the guidance of the respiratory and critical medicine departments. Alcoholor hydrogen peroxide-based rapid hand disinfectants are recommended. The aerosol generated by the electrotome should be gauged, and the machine should be used at the lowest effective power possible. The surgical specimens should be fixed and sealed in the negative pressure operating room and marked, and the inspection personnel should adopt secondary protection measures. Intraoperative irrigation should be reduced to avoid spillage of the patient's body fluid, and care should be taken to avoid accidental injury due to sharp instruments.

\section{Postoperative precautions}

For confirmed non-infected patients, the routine postoperative management process should be followed. Patients with suspected or confirmed new COVID-19 infection should be transported to the isolation ward through dedicated channels. Postoperative management should be jointly performed by the orthopedic and isolation ward physicians, and the results of the suspected cases should be tracked. Personnel involved in the operation should take off protective clothing, disinfect, bathe, and clean the cavity following standard procedures. Postoperative isolation for 14 days is also recommended, during which period the suitable medical treatment should be supplied if required. Double-layer yellow garbage bags should be used to seal the disposable articles used during the operation, and the articles contaminated with patient blood, body fluids, and secretions should be marked and treated as infectious medical waste. Anesthesia and surgical instruments should be disinfected or discarded in time. Operating rooms and transfer beds should be sprayed with disinfectant by intraoperative fluoroscopy machine and wiped regularly. Sampling tests should be conducted after disinfection, and the instruments should be used only after passing the standard requirements. The ventilation system filters in the operating room should be replaced on time.

\section{Postoperative management}

Supportive and nutritional treatment should be prioritized after the operation. Patients without COVID-19 infection can be treated in general wards. The confirmed cases should be transferred to the isolation ward, and the suspected cases should be isolated in a single room until the diagnosis is confirmed. With the focus on stabilizing the orthopedic condition, COVID-19 and its complications should be treated actively in cooperation with relevant departments. The traffic of medical personnel should be controlled, body temperature should be monitored, and serological tests should be carried out regularly.

Furthermore, any fever symptoms should be distinguished, and the patients should be observed for dyspnea and decreased oxygen saturation. Additionally, deep venous thrombosis of the lower extremities should be prevented, and anticoagulants can be used appropriately for high-risk patients. Patient blood samples, body fluids, secretions, excreta, and disposable personal belongings should be managed centrally.

\section{Discussion}

The number of fracture patients has decreased significantly during the epidemic compared to the same period the previous year. These results could be attributed to the general lack of activity due to the lockdown. Not surprisingly, the incidence of fractures caused by outdoor causes such as traffic accidents has decreased, while that due to home accidents, such as broken legs from falling, have increased. Also, the prevalence of female elderly hip fracture patients is significantly higher compared to their male counterparts, which could be due to menopause-related osteoporosis that significantly increases the risk of fractures. Furthermore, men are less likely to engage in manual labor during this period and therefore have a lower risk of hip fractures. The elderly should ensure a certain amount of activity every day and pay attention to the daily calcium intake, especially the older women engaged in manual labor. Regular physical check-ups to check bone density are also recommended, reducing the incidence of hip fractures.

Studies show that delayed surgery in elderly patients with hip fractures increases mortality rates, whereas timely surgery as permitted by the patient's physical condition can shorten hospitalization and reduce postoperative complications. At present, international guidelines advocate early surgical intervention for patients ( $<48$ hours). However, the waiting time for surgery in the outbreak period is $4.5+4$ days due to mandatory COVID-19 screening. Furthermore, many older people suffer from anemia and have an insufficient circulatory ability. Thus, the lower availability of blood in the clinics because the 
epidemic is another reason for delayed surgery.

Following the existing reports (16), fever (87.9\%) and cough $(67.7 \%)$ are the most common symptoms in patients with new coronavirus infection, while diarrhea $(3.7 \%)$ and vomiting ( $5 \%$ ) are less frequent. Therefore, body temperature screening is compulsory during outpatient preexamination, although it is not $100 \%$ accurate since many patients are asymptomatic. Since these missed patients can become "super spreaders," the medical history of suspected cases should be examined, and any symptoms or signs that cannot be explained by the primary disease should be recorded. Also, the key to controlling infectious diseases is to reduce personnel mobility and contact. Therefore, all hospitalized patients, regardless of the risk of contracting new corona infections, should be housed in single rooms to minimize contact with potentially infectious individuals. Separate isolation wards have therefore been set up for emergency trauma surgery, and full protection wards are implemented as buffer zones. For patients with significant trauma, life-threatening, or extremely threatening diseases that need emergency surgery, the isolation ward can be used as a postoperative treatment ward. These additions would allow for proper screening of patients with suspected COVID-19 while minimizing the risk of nosocomial infections. A temporary treatment site for suspected patients should also be set up for the emergency screening, and the patient should be transferred to the trauma surgical isolation ward immediately to reduce contact with other patients in the hospital. The confirmed cases can then be transferred to the designated hospital for COVID-19. Noninfected patients should be transferred to the corresponding treatment sites and treated so. By following these guidelines, no positive neocorona cases were detected in the medical personnel in our department.

There are several limitations in our study that ought to be considered. Since the data was collected separately, the omission of some case details was unavoidable. Besides, the missed diagnosis of fractures should also be considered. However, we believe that the missing data did not introduce any bias. Furthermore, high-energy injuries may also lead to other fractures, but we did not analyze the multiple fractures, or the underlying causes, treatment methods, and clinical outcomes of the fractures.

\section{Conclusions}

To conclude, the incidence of hip fractures among the elderly has increased during the COVID-19 epidemic. And the medical steps for the treatment of fracture patients during this period were formulated. However, mandatory screening has delayed surgical intervention by more than 48 hours. The orthopedic department should prioritize screening of emergency patients to minimize the risk of infection among other patients and medical personnel.

\section{Acknowledgments}

Funding: This study was supported in part by Shanghai Natural Science Foundation Program (19ZR1448000), the Youth Science and Technology Innovation Studio of Shanghai Jiaotong University School of Medicine (JYKCGZS01).

\section{Footnote}

Reporting Checklist: The authors have completed the STROBE reporting checklist. Available at http://dx.doi. org/10.21037/atm-20-4174

Data Sharing Statement: Available at http://dx.doi. org/10.21037/atm-20-4174

Conflicts of Interest: All authors have completed the ICMJE uniform disclosure form (available at http://dx.doi. org/10.21037/atm-20-4174). The authors have no conflicts of interest to declare.

Ethical Statement: The authors are accountable for all aspects of the work in ensuring that questions related to the accuracy or integrity of any part of the work are appropriately investigated and resolved. This study was approved by the ethics committee of our hospital (No. KY2020-125). All procedures performed in this study involving human participants were in accordance with the Declaration of Helsinki (as revised in 2013). Because of the retrospective nature of the research, the requirement for informed consent was waived.

Open Access Statement: This is an Open Access article distributed in accordance with the Creative Commons Attribution-NonCommercial-NoDerivs 4.0 International License (CC BY-NC-ND 4.0), which permits the noncommercial replication and distribution of the article with the strict proviso that no changes or edits are made and the original work is properly cited (including links to both the formal publication through the relevant DOI and the license). 
See: https://creativecommons.org/licenses/by-nc-nd/4.0/.

\section{References}

1. WHO. Novel coronavirus-China. Jan 12, 2020. Available online: http://www.who. int/csr/don/12-january-2020novel-coronavirus-china/en/ (accessed Jan 19, 2020).

2. Zhu N, Zhang D, Wang W, et al. A Novel Coronavirus from Patients with Pneumonia in China, 2019. N Engl J Med 2020;382:727-33.

3. Wang C, Horby PW, Hayden FG, et al. A novel coronavirus outbreak of global health concern. Lancet 2020;395:470-3.

4. General Office of National Health Commission of the People's Republic of China, Office of State Administration of Traditional Chinese Medicine of the People's Republic of China. Diagnosis and treatment scheme for novel coronavirus pneumonia (trial implementation 5th edition, revision edition). Available online: http://www.gov.cn/ zhengce/2020-02/09/content_5476415.htm

5. Yang Y, Lu Q, Liu M, et al. Epidemiological and clinical features of the 2019 novel coronavirus outbreak in China. MedRxiv 2020. doi: 10.1101/2020.02.10.20021675.

6. Wang D, Hu B, Hu C, et al. Clinical Characteristics of 138 Hospitalized Patients With 2019 Novel CoronavirusInfected Pneumonia in Wuhan, China. JAMA 2020;323:1061-9.

7. Li Q, Guan X, Wu P, et al. Early Transmission Dynamics in Wuhan, China, of Novel Coronavirus-Infected Pneumonia. N Engl J Med 2020;382:1199-207.

8. Huang C, Wang Y, Li X, et al. Clinical features of patients infected with 2019 novel coronavirus in Wuhan, China. Lancet 2020;395:497-506.

9. Shen MH, Yang CT, Wu CC, et al. Resuming Normal Life as a Family Caregiver During Drip-Like Recovery of Older Persons With Cognitive Impairment Recovering From Hip Surgery: A Grounded Theory. J Nurs Scholarsh 2020;52:250-60.

10. Hepping AM, Barvelink B, Ploegmakers JJW, et al. Recovery of strength after reduced pediatric fractures of the forearm, wrist or hand; A prospective study. PLoS One 2020;15:e230862.

11. Yang $Z, N i$ J, Long $Z$, et al. Is hip fracture surgery safe for patients on antiplatelet drugs and is it necessary to delay surgery? A systematic review and meta-analysis. J Orthop Surg Res 2020;15:105.

12. Meinberg EG, Agel J, Roberts CS, et al. Fracture and Dislocation Classification Compendium-2018. J Orthop Trauma 2018;32 Suppl 1:S1-170.

13. Lei J, Li J, Li X, et al. CT Imaging of the 2019 Novel Coronavirus (2019-nCoV) Pneumonia. Radiology 2020;295:18.

14. Wang XH, Pan ZY, Cheng ZS. Association between 2019$\mathrm{nCoV}$ transmission and $\mathrm{N} 95$ respirator use. MedRxiv 2020. doi: 10.1101/2020.02.18.20021881.

15. Tao K, Zhang B, Zhang P, et al. Recommendations for diagnosis, treatment, prevention and control of general surgery in the context of new coronavirus pneumonia. Chinese Journal of Surgery 2020;58:E001.

16. Guan WJ, Ni ZY, Hu Y, et al. Clinical characteristics of 2019 novel coronavirus infection in China. MedRxiv 2020. doi: 10.1101/2020.02.06.20020974.
Cite this article as: $\mathrm{Yu} \mathrm{P}, \mathrm{Wu} \mathrm{C}$, Zhuang $\mathrm{C}$, Ye T, Zhang $\mathrm{Y}$, Liu J, Wang L. The patterns and management of fractures under COVID-19 outbreak in China. Ann Transl Med 2020;8(15):932. doi: 10.21037/atm-20-4174 